\title{
PARIENTES SILVESTRES DEL TOMATE COMO FUENTE DE GERMOPLASMA PARA EL MEJORAMIENTO GENETICO DE LA ESPECIE
}

\section{TOMATO WILD RELATIVES AS A SOURCE OF GERMPLASM FOR BREEDING OF THE SPECIES}

\author{
Luis A. Flores-Hernández¹, Ricardo Lobato-Ortiz * J. Jesús García-Zavala', José \\ D. Molina-Galán', Dora Ma. Sargerman-Jarquín ${ }^{2}$ y Mario de J. Velasco-Alvarado ${ }^{1}$
}

\begin{abstract}
'Campus Montecillo, Postgrado de Recursos Genéticos y Productividad-Genética, Colegio de Postgraduados. Km. 36.5 Carr. México-Texcoco. 56230, Montecillo, Texcoco, Edo. De México. Tel. (595) 9520200 Ext. 1534. ${ }^{2}$ Campo experimental Valle de México, Instituto Nacional de Investigaciones Forestales, Agrícolas y Pecuarias. Km 13.5 Carr. Los Reyes-Texcoco. 56250, Coatlinchán, Texcoco, Edo. de México. A.P 10 y 307. Tel (595) 92 12681
\end{abstract}

*Autor para correspondencia (rlobato@colpos.mx)

\section{RESUMEN}

Debido a la reducida variabilidad genética de los cultivares modernos de tomate (Solanum lycopersicum L.), sus parientes silvestres son una fuente importante de genes de interés agronómico, como resistencia a factores bióticos y abióticos, base para el mejoramiento genético de la especie cultivada. El objetivo del estudio fue caracterizar morfológica y agronómicamente accesiones de especies silvestres de Solanum emparentadas con el tomate cultivado. Se evaluaron 46 accesiones de nueve especies silvestres en un diseño experimental completamente al azar en condiciones de invernadero. Se evaluaron 13 variables cuantitativas y cuatro cualitativas. Se encontraron diferencias significativas entre las especies para todas las variables y la comparación de medias mostró que estas diferencias están distribuidas en todas las especies. El análisis de conglomerados formó tres grupos claramente diferenciados. Las especies S. ochranthum y S. lycopersicoides fueron las de más alto contenido de sólidos, por lo que son promisorias para su uso en mejoramiento genético para este atributo de calidad.

Palabras clave: Solanum lycopersicum, parientes silvestres, recursos genéticos, mejoramiento genético.

\section{SUMMARY}

Due to reduced genetic variability of modern tomato (Solanum lycopersicum L.) cultivars, wild tomatoes are an important source for genes of agronomic interest. These traits include resistance to biotic and abiotic factors and provide a basis for the genetic improvement of the cultivated species. This study characterized morphological and agronomical traits from wild Solanum accessions related to cultivated tomato. Forty-six accessions of nine wild species of tomato were evaluated in a completely randomized experimental design under greenhouse conditions. Thirteen quantitative and four qualitative traits were evaluated. Significant differences were found among species for all the recorded traits, and mean comparisons showed that those differences were distributed across all species. Cluster analysis formed three clearly differentiated groups. S. lycopersicoides and S. ochranthum showed the highest solids content, hence both species are useful for improving genetically this quality trait in tomato.

Index words: Solanum lycopersicum, wild relatives, genetic resources, genetic improvement.

\section{INTRODUCCIÓN}

El tomate (Solanum lycopersicum L.) cultivado tiene una base genética muy estrecha, por lo que la variación den- tro y entre cultivares es muy pobre (Bai y Lindhout, 2007; García-Martínez et al., 2006; Miller y Tanksley, 1990); es por esta razón que los tomates silvestres son la fuente más importante de diversidad genética para usarse en el mejoramiento genético de la especie, pues en ellos se pueden encontrar genes de resistencia a factores causantes de estrés biótico y abiótico, amén de otros atributos de importancia agronómica (Bergougnoux, 2014; Foolad, 2007).

Los parientes silvestres del tomate cultivado se distribuyen a través de Ecuador, Perú, Chile y las Islas Galápagos (Peralta y Spooner, 2007), y México es considerado como centro de domesticación (Peralta y Spooner, 2007; Rick y Holle, 1990). Las especies silvestres tienen una amplia adaptación a diversos ambientes, debido a que crecen en diferentes altitudes, en montañas estrechas y geográficamente aisladas unas de otras, y también están adaptadas a condiciones microclimáticas específicas de suelo. Estas condiciones ecológicas han dado como resultado una amplia diversidad genética entre estas especies (Warnock, 1988)

Los parientes silvestres del tomate han sido ampliamente estudiados; se han realizado trabajos para conocer el origen del tomate cultivado (Bai y Lindhout, 2007; Bergougnoux, 2014), relaciones filogenéticas (Pease et al., 2016; Peralta y Spooner, 2000; Peralta y Spooner, 2001), y para la búsqueda de genes con características específicas en mejoramiento genético (Rick y Chetelat, 1995); sin embargo, no existen estudios realizados en México donde se hayan cultivado y caracterizado estas especies bajo condiciones de invernadero con el fin de usarlas en el mejoramiento genético de la especie cultivada. El objetivo del presente trabajo fue estudiar y describir la diversidad genética de especies silvestres emparentadas con el tomate cultivado, con potencial para ser aprovechadas en el mejoramiento genético de la especie cultivada en virtud de ser portadores de genes de importancia agronómica. 


\section{MATERIALES Y MÉTODOS}

El material vegetal estuvo constituido por 46 accesiones provenientes de nueve especies de tomates silvestres, las cuales fueron proporcionadas por el Tomato Genetics Resource Center (TGRC) de la Universidad de California, Davis, E.E.U.U. (Cuadro 1).

La evaluación de las accesiones se realizó en invernadero durante el ciclo primavera-verano 2014 en las instalaciones del Colegio de Postgraduados, Campus Motecillo, Texcoco, Estado de México (190 27' N, $98^{\circ} 54^{\prime}$ O, altitud de $2246 \mathrm{~m})$.

Las accesiones se evaluaron bajo un diseño experimental completamente al azar con cuatro repeticiones, una planta por bolsa y 10 plantas por unidad experimental. Las plantas se establecieron en bolsas negras de polietileno $(12 \mathrm{~L})$, con tezontle rojo como sustrato. La siembra se realizó en charolas de poliestireno con 200 cavidades el 28 de mayo de 2014 y las plántulas se trasplantaron 36 días después de la siembra (dds). Durante la etapa vegetativa las plantas se regaron con la solución nutritiva de Steiner (1984) al 25 \% de su concentración; en la floración y producción de fruto la concentración de la solución fue al 50 y $100 \%$, respectivamente. La solución de riego se mantuvo a un $\mathrm{pH}$ de 5.5 a 5.8. Las plantas se tutoraron con el objeto de mantenerlas erectas. Las plagas y enfermedades se controlaron con el uso de productos químicos Confidor ${ }^{\circledR}$ y Ampligo ${ }^{\circledR}$ para mosca blanca (Bemisia tabaci Gennadius), Captan $\AA$ y Ridomil Gold $®$ para tizón tardío (Phytophthora infestans) y Amistar ${ }^{\circledR}$ para tizón temprano (Altenaria solani).

Se midieron 13 variables cuantitativas: días a floración (DF), días a madurez (DM), longitud de hoja en cm (LH), ancho de hoja en $\mathrm{cm}(\mathrm{AH})$, diámetro de tallo en $\mathrm{cm}$ medido a los $5 \mathrm{~cm}$ de la base de la planta (DT), número de flores por racimo (FR), longitud del racimo en $\mathrm{cm}(\mathrm{LR})$, peso de fruto en $\mathrm{g}(\mathrm{PF})$, longitud (LF) y ancho de fruto (AF) en $\mathrm{cm}$, sólidos solubles totales (B), número de semillas por fruto (SF), y tipo de inflorescencia (TI). Los sólidos solubles se midieron con un refractómetro digital marca Atago® modelo PAL-1 (Tokio, Japón) con un rango de 0.0 a $53.0^{\circ}$ Brix. Las variables cualitativas fueron posición del estilo (PE), forma predominante del fruto (FP), rayas verdes en el fruto (RV) y pubescencia del fruto (PF); éstas fueron evaluadas de acuerdo con el manual de descriptores para tomate (S. lycopersicum L.) del Instituto Internacional de Recursos Fitogenéticos (IPGRI, 1996) y la guía de la Unión Internacional para la Protección de las Obtenciones Vegetales para tomate (UPOV, 2011)

Se realizaron análisis de varianza y comparación de medias (Tukey, $P \leq 0.05$ ) a los datos de las variables cuantitativas. También se realizó un análisis de componentes principales (CP) mediante la matriz de correlaciones (SAS Institute, 2002) y se graficaron los datos de los componentes principales. Por último, se hizo un análisis de conglomerados con la distancia euclidiana (método de agrupamiento UPGMA) con el paquete estadístico $R$ ( $R$ Development Core Team, 2012), con la finalidad de agrupar las colectas en relación con las distancias euclidianas.

\section{RESULTADOS Y DISCUSIÓN}

El análisis de varianza detectó significancia $(P \leq 0.01)$ entre especies, entre accesiones, y en el anidamiento accesiones dentro de especies, para todas las variables (Cuadro 2). Los coeficientes de variación más altos fueron para peso de fruto (55\%), número de semillas por fruto (28\%), número de flores por racimo (27\%), longitud del racimo (26\%), tipo de inflorescencia (23\%), y días a floración (21\%), mientras que el resto de las variables tuvieron

Cuadro 1. Especies silvestres de Solanum (Peralta et al., 2008) utilizadas en el estudio, accesiones y país de origen.

\begin{tabular}{lll}
\hline Especie & Accesión & País de origen \\
\hline S. pennellii & LA 2580, LA 0716, LA 1272, LA 1367, LA 1277. & Perú \\
S. pimpinellifollium & LA 1584, LA 1689, LA 1237, LA 1593, LA 1576, LA 0373, LA 0442. & Perú \\
S. peruvianum & LA 2172, LA 1982, LA 1677, LA 1973, LA 1360, LA 0103, LA 0446, LA 1346, LA & Perú \\
S. neorickii & 1336, LA 1274, LA 1365, LA 2152. & Perú \\
S. lycopersicoides & LA 2319, LA 2133. & Chile \\
S. habrochaites & LA 2408. & Perú, Ecuador \\
S. chmielewski & LA 2409, LA 1731, LA 2650, LA 2158, LA 2167, LA 1223, LA 1777, GH0810. & Perú \\
S. ochranthum & LA 2162. & Perú \\
S. chilense & LA 2930, LA 1960, LA 2759, LA 1958, LA 2778, LA 2748, LA 2750. & Chile, Perú \\
\hline
\end{tabular}


coeficientes de variación menores a 20 \%. Dado que las especies se evaluaron bajo las mismas condiciones, gran parte de la variabilidad que existe entre y dentro de ellas se debe a su tipo de hábitat estrecho y aislado, lo que significa que existe una adaptación a diferentes tipos de suelo y microclimas particulares (Bauchet y Causse, 2012), pero también se debe a diferencias genéticas intrínsecas dentro de cada una de ellas (Peralta y Spooner, 2005).

La prueba de medias para las 13 variables cuantitativas (Cuadro 3 ) indica que la especie más precoz fue $S$. pimpinellifollium (14 d), mientras que la más tardía fue $S$. ochranthum (102 d). En relación con lo anterior, los diferentes hábitats de adaptación de las especies evaluadas han dado como resultado diferencias en el tiempo a la floración, debido a que sus nichos ecológicos son contrastantes, y además proporcionan oportunidad de diversificarse rápidamente y adaptarse a sus entornos locales (Moore y Donoghue, 2007). Los días a madurez de fruto entre las especies también fueron muy contrastantes, por la alta correlación entre esta variable y los días a la floración. Las diferencias tan grandes para la floración y maduración de frutos de $S$. ochranthum con las demás especies se deben a que esta especie es considerada una de las más alejadas filogenéticamente del tomate cultivado y de S. pimpinellifollium, la que a su vez es considerada como una de las especies silvestres más cercanas al tomate cultivado (Peralta y Spooner, 2001).

En longitud de hoja contrastan los valores de S. lycopersicoides $(13 \mathrm{~cm})$ con los de S. ochranthum $(42.3 \mathrm{~cm})$ y S. habrochaites $(36.5 \mathrm{~cm})$; mientras que para ancho de hoja, el valor de S. lycopersicoides fue de $10.6 \mathrm{~cm}$ y el de S. ochranthum fue de $27.3 \mathrm{~cm}$. Esta misma especie tuvo el mayor diámetro de tallo $(1.76 \mathrm{~cm})$ y el más leñoso, en comparación con las otras especies, mientras que $S$. neorickii fue la de menor diámetro $(0.65 \mathrm{~cm})$. Estas características son producto de la evolución y diversificación que han desarrollado las especies al sobrevivir en condiciones extremas y tolerar factores bióticos y abióticos adversos (Eigenbrode et al., 1993).

Las especies S. pennellii, S. lycopersicoides, S. pimpineIlifollium, S. habrochaites y S. chilense estadísticamente tuvieron igual diámetro de tallo. Por otro lado, la especie con mayor número de flores por racimo fue $\mathrm{S}$. ochranthum, con 41 flores, y la de menor número fue S. chmielewski con 8 flores. Las especies S. chilense, S. habrochaites, S. peruvianum y $S$. pimpinellifollium tuvieron estadísticamente igual número de flores, lo mismo ocurrió entre S. pimpineIlifollium y S. pennellii. En cuanto al tipo de inflorescencia, la especie S. ochranthum fue multípara con el mayor número

Cuadro 2. Cuadrados medios de las fuentes de variación, intervalos, promedios y desviación estándar de 13 variables cuantitativas evaluadas en nueve especies silvestres de tomate (Solanum spp.).

\begin{tabular}{|c|c|c|c|c|c|c|c|c|}
\hline \multirow[b]{2}{*}{ Variable } & \multicolumn{4}{|c|}{ Fuentes de variación } & \multirow[b]{2}{*}{ CV (\%) } & \multirow[b]{2}{*}{ Intervalo } & \multirow[b]{2}{*}{ Media } & \multirow[b]{2}{*}{$\mathrm{DE}$} \\
\hline & Especie & $\begin{array}{l}\text { Accesión } \\
\text { (especie) }\end{array}$ & Accesión & Error & & & & \\
\hline DF & $3724 * *$ & $123 * \star$ & $728 * *$ & 39 & 21 & $10-02$ & 29 & 16 \\
\hline DM & $11,400 * *$ & $136 * *$ & $2141 * *$ & 41 & 7.5 & $49-247$ & 86 & 27 \\
\hline $\mathrm{LH}$ & $800 * *$ & $41 * *$ & $177 \star *$ & 17 & 17 & $12-50$ & 24 & 8 \\
\hline $\mathrm{AH}$ & $246 * *$ & $15 * \star$ & $59 * *$ & 7 & 20 & $7.5-29$ & 14 & 5 \\
\hline DT & $0.59 * *$ & $0.06 * *$ & $0.16 * \star$ & 0.2 & 15 & $0.6-1.9$ & 0.95 & 0.23 \\
\hline $\mathrm{FR}$ & $570 * *$ & $69 * *$ & $157 \star \star *$ & 25 & 27 & $7-45$ & 18 & 7.5 \\
\hline $\mathrm{Tl}$ & $11 * *$ & $0.37 * *$ & $2.2 * \star$ & 0.2 & 23 & $1-6$ & 1.8 & 0.9 \\
\hline LR & $714 \star \star$ & $100 * \star$ & $207 \star \star$ & 32 & 26 & $7-49$ & 22 & 8.5 \\
\hline $\mathrm{PF}$ & 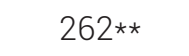 & $5.8 * \star$ & $7.1 * \star$ & 1.6 & 55 & $0.2-9.8$ & 2.3 & 1.5 \\
\hline LF & $3.1 * *$ & $0.12 * *$ & $0.65 * *$ & 0.04 & 14.5 & $0.75-4.2$ & 1.4 & 0.47 \\
\hline $\mathrm{AF}$ & $2.1 * *$ & $0.17 \star *$ & $0.51 * *$ & 0.05 & 14.5 & $0.78-3.6$ & 1.6 & 0.41 \\
\hline B & $11 * *$ & $4.6 * *$ & $5.7 \star \star$ & 1.8 & 16.5 & $5.3-11.5$ & 7.2 & 1.5 \\
\hline SF & $44,822 * *$ & $2083 * *$ & $9656 * *$ & 604 & 28 & $3-258$ & 90 & 57 \\
\hline $\mathrm{GL}$ & 8 & 36 & 45 & 93 & & & & \\
\hline
\end{tabular}

$\star \star P \leq 0.01 ;$ DE: Desviación estándar; DF: días a floración; DM: días a madurez; LH: Iongitud de hoja (cm); AH: ancho de hoja (cm); DT: diámetro de tallo (cm); FR: número de flores por racimo; TI: tipo de inflorescencia; LR: longitud de racimo (cm); PF: peso de fruto (g); LF: longitud de fruto (cm); AF: ancho de fruto (cm); B: sólidos solubles totales ( ${ }^{\circ}$ Brix); SF: número de semillas por fruto. 
de inflorescencias, mientras que especies como S. pimpinellifollium, S. neorickii y S. chmielewski fueron uníparas. Con respecto a la longitud del racimo, $S$. peruvianum y $S$. chilense tuvieron los racimos más grandes $(29$ y $28 \mathrm{~cm}$, respectivamente), mientras que S. neorickii y S. pimpinellifollium fueron las de menor longitud (12 y $14 \mathrm{~cm}$, respectivamente). Con respecto al peso de fruto, $\mathrm{S}$. peruvianum tuvo los frutos de mayor peso ( $3.4 \mathrm{~g})$, mientras que los frutos de $\mathrm{S}$. lycopersicoides tuvieron el menor peso $(0.2 \mathrm{~g})$, lo cual fue congruente con su longitud y diámetro de fruto reducidos. La especie con los frutos de mayor tamaño en longitud y ancho fue S. ochranthum, aunque el peso de sus frutos no fue el más alto, porque presenta una cavidad en el interior del fruto. Para el tamaño del fruto se ha señalado que la transición evolutiva del tomate silvestre a domesticado se basó en mutaciones asociadas con el aumento del tamaño de fruto, y que fueron seleccionadas y acumuladas por los agricultores (Bai y Lindhout, 2007).

Las especies S. ochranthum y S. lycopersicoides fueron las de más alto contenido de sólidos solubles totales; sin embargo, estas especies no presentan polinización cruzada con el tomate cultivado (Chetelat et al., 1997; Rick et al., 1990), por lo que se han estudiado estrategias para superar esta barrera reproductiva; una de ellas es modificar la técnica de polinización mediante una especie silvestre mejorada (S. pennellii) para la transferencia de genes a través de hibridación somática (Gradziel y Robinson, 1989; Kobayashi et al., 1996). La fusión de protoplastos en S. Iycopersicoides se ha realizado con éxito para la transferencia de genes (Hossain et al., 1994; Moore y Sink, 1988), lo que facilita la generación de híbridos fértiles.
Con respecto a la comparación de medias de las variables cualitativas (Cuadro 4), la forma predominante de los frutos (FP) fue diferente entre las especies, donde las de mayor frecuencia fueron ligeramente achatado ( $L$ ach) y achatado (ach); otras formas como redondo (Red) y redondo alargado (R-al) aparecieron en S. pimpinellifollium y $S$. ochranthum, respectivamente. Las especies $S$. peruvianum y S. neorickii presentaron frutos con estrías verdes (RV) y S. habrochaites con alta pubescencia (PF). Con respecto a la posición del estilo (PE), las especies S. chilense, S. neorickii y S. chmielewski presentaron el estilo inserto; S. pimpinellifollium y S. peruvianum mostraron estilo tanto inserto como exerto. El resto de las especies tuvieron un estilo exerto. En este sentido, Bergougnoux (2014) indica que las especies con el estilo exerto son autoincompatibles y presentan mayor grado de diversidad genética debido a que el estigma y estilo exerto así como la autoincompatibilidad del gametofito contribuyen a una mayor polinización cruzada, lo que equivale a mayor diferenciación genética, a diferencia de las especies autocompatibles, que se caracterizan por poseer baja diversidad, con flores pequeñas y un estilo inserto.

En el análisis de componentes principales, la matriz de valores propios mostró que los tres primeros componentes concentraron $73 \%$ de la variación (Cuadro 5), donde el primer componente principal (CP 1) explicó el $49 \%$ de la variación. Las variables relacionadas con el inicio de la floración y las asociadas al rendimiento de fruto fueron las que contribuyeron en mayor proporción a la varianza explicada. Bonilla-Barrientos et al. (2014) señalan a estas variables como las de mayor varianza en la caracterización de tomate tipo pimiento y arriñonado. Por su parte,

Cuadro 3. Comparación de medias para 13 variables de nueve especies silvestres de tomate (Solanum spp.).

\begin{tabular}{lllllllllllllll}
\hline Especie & DF & DM & LH & AH & DT & FR & TI & LR & PF & LF & AF & B & SF \\
\hline pen & $33 \mathrm{bcd}$ & $89 \mathrm{bc}$ & $20.5 \mathrm{bc}$ & $12.4 \mathrm{c}$ & $1.19 \mathrm{~b}$ & $14 \mathrm{e}$ & $2 \mathrm{de}$ & $25 \mathrm{a}$ & $2.6 \mathrm{ab}$ & $1.3 \mathrm{~b}$ & $1.8 \mathrm{~b}$ & $7.9 \mathrm{ab}$ & $197 \mathrm{a}$ \\
pim & $14 \mathrm{e}$ & $63 \mathrm{~d}$ & $19.3 \mathrm{bcd}$ & $11.8 \mathrm{c}$ & $0.98 \mathrm{bc}$ & $16 \mathrm{cde}$ & $1 \mathrm{e}$ & $14 \mathrm{c}$ & $1.4 \mathrm{abc}$ & $1.2 \mathrm{~b}$ & $1.3 \mathrm{c}$ & $7 \mathrm{~b}$ & $27 \mathrm{ef}$ \\
per & $19 \mathrm{e}$ & $83 \mathrm{bcd}$ & $21.8 \mathrm{bc}$ & $12.5 \mathrm{c}$ & $0.80 \mathrm{~cd}$ & $19 \mathrm{~cd}$ & $2 \mathrm{~d}$ & $29 \mathrm{a}$ & $3.4 \mathrm{a}$ & $1.4 \mathrm{~b}$ & $1.6 \mathrm{bc}$ & $6.6 \mathrm{~b}$ & $123 \mathrm{~b}$ \\
neo & $24 \mathrm{de}$ & $82 \mathrm{~cd}$ & $19.8 \mathrm{bc}$ & $12.9 \mathrm{c}$ & $0.65 \mathrm{~d}$ & $9 \mathrm{e}$ & $1 \mathrm{e}$ & $12 \mathrm{c}$ & $1.3 \mathrm{bc}$ & $1.2 \mathrm{~b}$ & $1.4 \mathrm{c}$ & $6.7 \mathrm{~b}$ & $98 \mathrm{bc}$ \\
lyc & $39 \mathrm{bc}$ & $77 \mathrm{~d}$ & $13 \mathrm{~d}$ & $10.6 \mathrm{c}$ & $1.13 \mathrm{~b}$ & $31 \mathrm{~b}$ & $3 \mathrm{~b}$ & $16 \mathrm{c}$ & $0.2 \mathrm{c}$ & $0.8 \mathrm{c}$ & $0.8 \mathrm{~d}$ & $9.5 \mathrm{a}$ & $4 \mathrm{f}$ \\
hab & $43 \mathrm{~b}$ & $93 \mathrm{~b}$ & $36.5 \mathrm{a}$ & $20.8 \mathrm{~b}$ & $0.97 \mathrm{bc}$ & $22 \mathrm{~cd}$ & $2 \mathrm{~cd}$ & $17 \mathrm{c}$ & $2.9 \mathrm{ab}$ & $1.4 \mathrm{~b}$ & $1.7 \mathrm{bc}$ & $8 \mathrm{ab}$ & $84 \mathrm{bcd}$ \\
chm & $33 \mathrm{bcd}$ & $83 \mathrm{bcd}$ & $18.2 \mathrm{~cd}$ & $11.2 \mathrm{c}$ & $0.78 \mathrm{~cd}$ & $8 \mathrm{e}$ & $1 \mathrm{e}$ & $15 \mathrm{c}$ & $1.5 \mathrm{abc}$ & $1.3 \mathrm{~b}$ & $1.5 \mathrm{bc}$ & $8 \mathrm{ab}$ & $70 \mathrm{~cd}$ \\
och & $102 \mathrm{a}$ & $247 \mathrm{a}$ & $42.3 \mathrm{a}$ & $27.3 \mathrm{a}$ & $1.76 \mathrm{a}$ & $41 \mathrm{a}$ & $6 \mathrm{a}$ & $20 \mathrm{abc}$ & $2.8 \mathrm{ab}$ & $4.1 \mathrm{a}$ & $3.5 \mathrm{a}$ & $9.7 \mathrm{a}$ & $55 \mathrm{de}$ \\
chil & $32 \mathrm{~cd}$ & $86 \mathrm{bcd}$ & $25.7 \mathrm{~b}$ & $15.2 \mathrm{~b}$ & $1.02 \mathrm{bc}$ & $22 \mathrm{c}$ & $3 \mathrm{bc}$ & $28 \mathrm{a}$ & $1.5 \mathrm{abc}$ & $1.2 \mathrm{~b}$ & $1.4 \mathrm{c}$ & $6.5 \mathrm{~b}$ & $51 \mathrm{de}$ \\
DSH & 10 & 10.4 & 6.7 & 4.8 & 0.23 & 8 & 0.7 & 9 & 2.1 & 0.32 & 1.21 & 1.9 & 41 \\
\hline
\end{tabular}

pen: S. pennellii; pim: S. pimpinellifollium; per: S. peruvianum; neo: S. neorickii; lyc: S. lycopersicoides; hab: S. habrochaites; chm: S. chmielewski; och: S. ochranthum; chil: S. chilense; DSH: diferencia significativa honesta; DF: días a floración; DM: días a madurez; LH: longitud de hoja (cm); AH: sncho de hoja (cm); DT: diámetro de tallo (cm); FR: número de flores por racimo; Tl: tipo de inflorescencia; LR: longitud de racimo (cm); PF: peso de fruto (g); LF: longitud de fruto (cm); AF: ancho de fruto (cm); B: sólidos solubles totales ( ${ }^{\circ}$ Brix); SF: número de semillas por fruto. Medias con letra igual dentro de columnas no son estadísticamente diferentes (Tukey, $\mathrm{P} \leq 0.05$ ). 
Cuadro 4. Variables cualitativas evaluadas en los parientes silvestres del tomate (Solanum spp.).

\begin{tabular}{lcccc}
\hline Especie & FP & RV & PF & PE \\
\hline S. pennellii & Ach & Aus & Inter & Ex \\
S. pimpinellifollium & Red & Aus & Esc & Ex/ln \\
S. peruvianum & L ach & Pres & Esc & Ex/ln \\
S. neorickii & Ach & Pres & Esc & In \\
S. lycopersicoides & Ach & Aus & Esc & Ex \\
S. habrochaites & Lach & Pres/Aus & Den & Ex \\
S. chmielewski & Lach & Aus & Esc & In \\
S. ochranthum & R-al & Aus & Esc & Ex \\
S. chilense & Lach/Ach & Aus & Inter/Esc & In \\
\hline
\end{tabular}

FP. forma predominante del fruto; RV: rayas verdes en el fruto; PF: pubescencia en el fruto; PE: posición del estilo; Ach: achatado; L ach: ligeramente achatado; Red: redondo; R-al: redondo-alargado; Aus: ausente; Pres: presente; Esc: escasa; Inter: intermedia; Den: densa; In: inserto; Ex: exerto.

Ríos-Osorio et al. (2014), al evaluar cambios biofísicos y la variabilidad de frutos del tomate, indican que la variable peso de fruto aportó la mayor varianza.

El segundo componente principal (CP 2) explicó el $13 \%$ de la variación, y las variables fenológicas, así como el número de semillas, fueron las que más influyeron en la variabilidad de este segundo componente. El tercer componente principal (CP 3) explicó el 11 \% de la variación, donde la variable sólidos solubles, relacionada con la calidad, y la variable relacionada con la precocidad (días a madurez), fueron las que más influyeron en la variación. Estos resultados concuerdan con los de Moya et al. (2005), quienes realizaron el análisis de componentes principales para la selección y evaluación de nuevas líneas y variedades de tomate de crecimiento indeterminado con altos rendimientos y calidad de fruto, donde el peso de fruto, altura de planta, diámetro de fruto y días a madurez explicaron la mayor variabilidad, lo que indica que tanto las especies silvestres como la cultivada tienen una estructura similar de variabilidad.

Cuadro 5. Valores propios de los tres primeros componentes principales (CP).

\begin{tabular}{lccc}
\hline Variables & CP 1 & CP 2 & CP 3 \\
\hline Días a floración & -0.330 & -0.191 & 0.015 \\
Días a madurez & -0.365 & 0.067 & 0.031 \\
Longitud de hoja (cm) & -0.27 & -0.303 & -0.302 \\
Ancho de hoja (cm) & -0.286 & -0.346 & -0.257 \\
Diámetro de tallo (cm) & -0.231 & -0.058 & 0.011 \\
Número de flores por racimo & -0.273 & -0.004 & -0.267 \\
Tipo de inflorescencia & -0.333 & 0.038 & -0.110 \\
Longitud de racimo (cm) & -0.043 & 0.486 & -0.566 \\
Peso de fruto (g) & -0.349 & 0.170 & 0.194 \\
Longitud de fruto (cm) & -0.343 & 0.162 & 0.227 \\
Ancho de fruto (cm) & -0.320 & 0.280 & 0.196 \\
Sólidos solubles totales ( ${ }^{\circ}$ Brix) & -0.112 & -0.145 & 0.547 \\
Número de semillas por fruto & -0.024 & 0.591 & 0.090 \\
Varianza explicada (\%) & 48.69 & 13.07 & 10.84 \\
Varianza acumulada (\%) & 48.69 & 61.76 & 72.60 \\
\hline
\end{tabular}

CP. componente principal. 
En la Figura 1 se representan los tres primeros componentes principales, que explicaron el $73 \%$ del total de la variación. Las variables longitud y ancho de hoja fueron las que registraron mayor varianza en el componente 1 (CP 1); en el componente 2 (CP 2) la mayor varianza se relacionó con los sólidos solubles totales, así como con la longitud del racimo; en el componente 3 (CP 3) el número de semillas por fruto registró el nivel más alto de varianza. Las variables que aportaron mayor variación concuerdan con las reportadas por Morales et al. (1996) al avaluar la variabilidad de somaclones generados a partir de una población de tomate. Con base en el análisis de componentes principales, se realizó un análisis de conglomerados con los tres primeros componentes que determinaron el más alto porcentaje de variación. En la Figura 2 se muestra el dendrograma resultado del análisis de conglomerados, donde se formaron tres grupos $(A, B, C)$.

El grupo A estuvo integrado por las especies: S. pennellii, S. peruvianum, S. chilense y dos accesiones de S. pimpineIlifollium (LA 1584, LA 1689). Las especies S. peruvianum y S. chilense están estrechamente relacionadas (Städler et al., 2008), pues ambas especies presentan autoincompatibilidad (Peralta y Spooner, 2000), y su hábitat se encuentra a 3000 msnm, lo que las hace parecidas. S. pennellii es una especie que generalmente es autoincompatible, aunque la compatibilidad se puede encontrar en algunas accesiones (e.g. LA 0716); por lo tanto, el grupo quedó definido por las especies que presentan autoincompatibilidad, además de otras características como el tamaño y color de los frutos
(RV), que fueron similares para estas especies, como se observa en el Cuadro 4.

El grupo B estuvo integrado por las accesiones de las especies S. pimpinellifollium, S. lycopersicoides, S. chmielewski y S. neorickii, donde esta última presentó gran similitud con el tomate cultivado (Nakazato y Housworth, 2011). Las cuatro especies que formaron este grupo muestran autocompatibilidad (Peralta y Spooner, 2001). S. Iycopersicoides (LA 2408) se ubicó en este grupo, porque comparte características morfológicas, como la longitud y el ancho de las hojas. Bretó et al. (1993), mediante marcadores enzimáticos, demostraron que las especies $S$. chmielewski, S. neorickii y S. pimpinellifollium presentan una amplia diversidad, incluso mayor que otras especies silvestres. Rick (1983) realizó un estudio de diversidad genética en las especies silvestres con marcadores enzimáticos, en donde encontró que S. chmielewski y S. neorickii están muy emparentadas, con una gran cantidad de genes en común.

La especie S. habrochaites formó el grupo C. Esta especie, al igual que las del grupo A, se caracteriza por presentar autoincompatibilidad; sin embargo, aspectos como la pubescencia en tallos y frutos, el tipo de crecimiento de la planta y su nicho ecológico contrastan con las otras especies autoincompatibles (Bergougnoux, 2014). Nakazato y Housworth (2011) estudiaron la influencia geográfica andina en la especiación de los parientes silvestres del tomate cultivado, y afirman que las condiciones ecológicas son

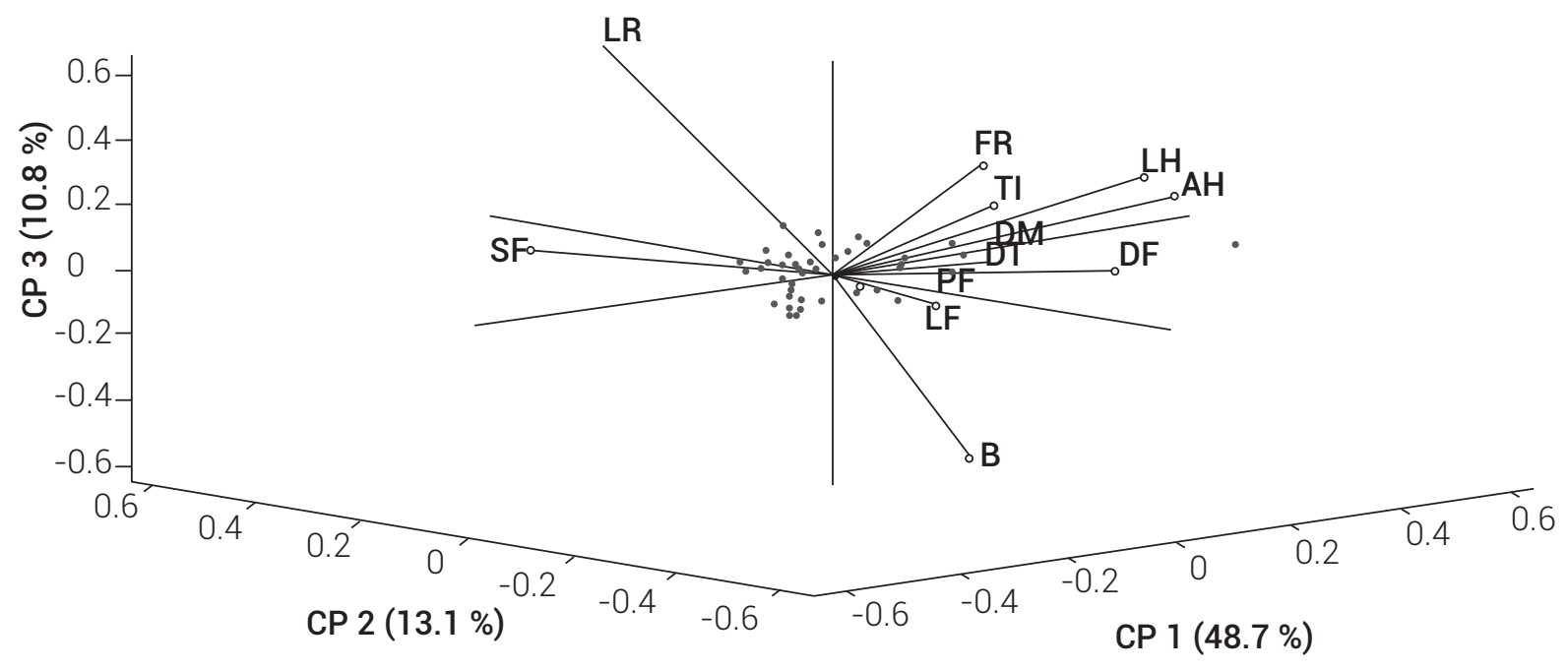

Figura 1. Dispersión de las 46 accesiones de las especies silvestres, en el plano determinado por los tres primeros componentes principales (CP). DF: días a floración del primer racimo; DM: días a maduración del primer racimo; $\mathrm{LH}$ : longitud de la hoja (cm); AH: ancho de la hoja $(\mathrm{cm})$; DT: diámetro de tallo $(\mathrm{cm})$; FR: número de flores por racimo; TI: tipo de inflorescencia; LR: longitud del racimo $(\mathrm{cm})$; PF: peso de fruto (g); LF: longitud de fruto $(\mathrm{cm})$; AF: ancho de fruto $(\mathrm{cm})$; B: sólidos solubles totales ( ${ }^{\circ}$ Brix); SF: número de semillas por fruto. 


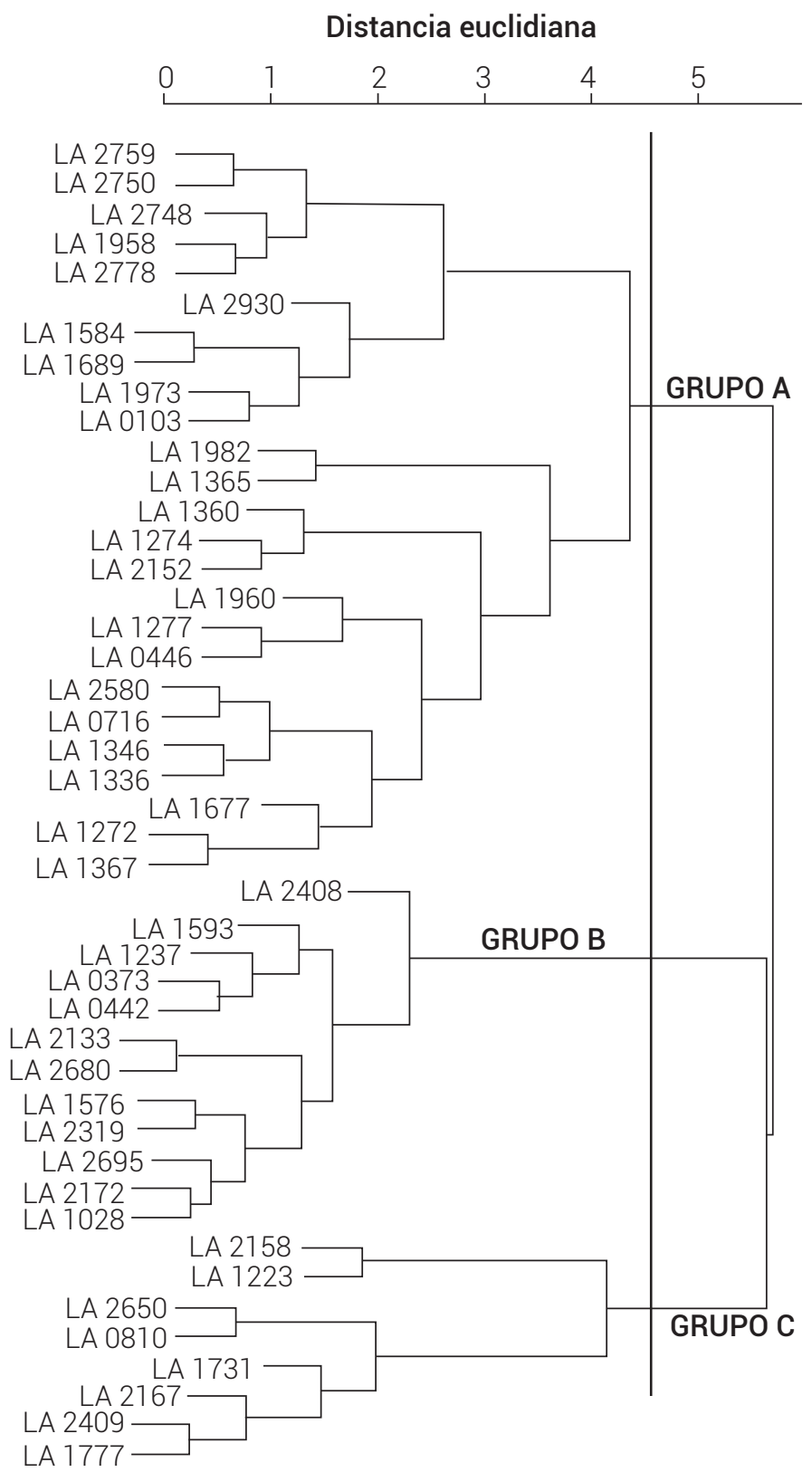

Figura 2. Dendrograma de 45 accesiones de parientes silvestres de tomate (Solanum spp.) mediante el agrupamiento UPGMA y las distancias euclidianas derivadas en 13 variables morfológicas.

fundamentales para la evolución fenotípica y especiación, lo que determina la formación de grupos genéticos con rasgos compartidos.

\section{CONCLUSIONES}

Cada una de las especies silvestres estudiadas tiene características potenciales para ser usadas en el mejoramiento genético del tomate cultivado. Las variables relacionadas con los componentes del rendimiento, precocidad y sólidos solubles totales son factores importantes en la definición de las especies silvestres a utilizar para mejorar genéticamente al tomate cultivado.

Entre las especies, las variables de rendimiento, precocidad y sólidos solubles influyeron en mayor medida en la variabilidad total expresada.

Las accesiones de las especies silvestres se agruparon por especies, de las cuales se formaron tres grupos genéticos, donde la autoincompatibilidad, la pubescencia y las variables evaluadas permitieron una diferenciación clara 
de los grupos.

La diversidad genética de los parientes silvestres del tomate estudiados está distribuida entre y dentro de especies, por lo que representan una fuente de variación importante para usarse en el mejoramiento de las actuales variedades de tomate, para aumentar la producción y el valor nutricional.

Las especies S. ochranthum y S. lycopersicoides fueron las de más alto contenido de sólidos solubles por lo que son promisorias para su uso en mejoramiento genético para este atributo en el tomate cultivado.

\section{BIBLIOGRAFÍA}

Bai Y. and P. Lindhout (2007) Domestication and breeding of tomatoes: what have we gained and what can we gain in the future? Annals of Botany 100:1085-1094.

Bauchet G. and M. Causse (2012) Genetic diversity in tomato (Solanum lycopersicum) and its wild relatives. In: Genetic Diversity in Plants. M. Caliskan (ed.). InTech. Rijeka, Croatia. pp:133-162.

Bergougnoux V. (2014) The history of tomato: from domestication to biopharming. Biotechnology Advances 32:170-189.

Bonilla-Barrientos 0., R. Lobato-Ortiz, J. J. García-Zavala, S. Cruz-Izquierdo, D. Reyes-López, E. Hernández-Leal y A. Hernández-Bautista (2014) Diversidad agronómica y morfológica de tomates arriñonados y tipo pimiento de uso local en Puebla y Oaxaca, México. Revista Fitotecnia Mexicana 37:129-139.

Bretó M. P., M. J. Asins and E. A. Carbonell (1993) Genetic variability in Lycopersicon species and their genetic relationships. Theoretical and Applied Genetics 86:113-120.

Chetelat R. T., P. Cisneros, L. Stamova and C. M. Rick (1997) A male-fertile Lycopersicon esculentum $\times$ Solanum lycopersicoides hybrid enables direct backcrossing to tomato at the diploid level. Euphytica 95:99-108.

Eigenbrode S. D., J. T. Trumble and R. A. Jones (1993) Resistance to beet armyworm, Hemipterans, and Liriomyza spp. in Lycopersicon accessions. Journal of the American Society for Horticultural Science 118:525-530.

Foolad M. R. (2007) Genome mapping and molecular breeding of tomato. International Journal of Plant Genomics 52 p. http://dx.doi. org/10.1155/2007/64358

García-Martínez S., L. Andreani, M. Garcia-Gusano, F. Geuna and J. J. Ruiz (2006) Evaluation of amplified fragment length polymorphism and simple sequence repeats for tomato germplasm fingerprinting: utility for grouping closely related traditional cultivars. Genome 49:648-656.

Gradziel T. M. and R. W. Robinson (1989) Solanum lycopersicoides gene introgression to tomato, Lycopersicon esculentum, through the systematic avoidance and suppression of breeding barriers. Sexual Plant Reproduction 2:43-52.

Hossain M., S. Imanishi and A. Matsumoto (1994) Production of somatic hybrids between tomato (Lycopersicon esculentum) and night shade (Solanum lycopersicoides) by electrofusion. Breeding Science 44:405-412.

IPGRI, Instituto Internacional de Recursos Fitogenéticos (1996) Descriptores para el Tomate (Lycopersicon spp.). Instituto Internacional de Recursos Fitogenéticos. Roma, Italia. 49 p.

Kobayashi R. S., J. R. Stommel and S. L. Sinden (1996) Somatic hybridization between Solanum ochranthum and Lycopersicon esculentum. Plant Cell, Tissue and Organ Culture 45:73-78.

Miller J. C. and S. D. Tanksley (1990) RFLP analysis of phylogenetic relationships and genetic variation in the genus Lycopersicon. Theoretical and Applied Genetics 80:437-448.

Moore B. R. and M. J. Donoghue (2007) Correlates of diversification in the plant clade Dipsacales: geographic movement and evolutionary innovations. The American Naturalist 170:S28-S55.
Moore P. P. and K. C. Sink (1988) Characterization of a Lycopersicon es culentum $\times$ Solanum lycopersicoides somatic hybrid lacking a glutamate oxaloacetate transaminase isozyme. Plant Cell, Tissue and Organ Culture 13:39-47.

Morales C., N. Santana, X. Xiqués, I. Reynaldo, B. Martínez, S. Peréz, M. I. Román y J. Rodríguez (1996) Variabilidad en somaclones de tomate provenientes del cultivar Campbell-28. Cultivos Tropicales 17:67-71.

Moya C., M. Álvarez, D. Plana, M. Florido y C. J. B. Lawrence (2005) Evaluación y selección de nuevas líneas de tomate (Lycopersicon esculentum Mill.) con altos rendimientos y frutos de alta calidad. Cultivos Tropicales 26:39-43.

Nakazato T. and E. A. Housworth (2011) Spatial genetics of wild tomato species reveals roles of the Andean geography on demographic history. American Journal of Botany 98:88-98.

Pease J. B., D. C. Haak, M. W. Hahn and L. C. Moyle (2016) Phylogenomics reveals three sources of adaptive variation during a rapid radiation. PLoS Biology 14:e1002379http://dx.doi.org/10.1371/ journal.pbio.1002379

Peralta I. E. and D. M. Spooner (2000) Classification of wild tomatoes: a review. Kurtziana 28:45-54.

Peralta I. E. and D. M. Spooner (2001) Granule-bound starch synthase (GBSSI) gene phylogeny of wild tomatoes (Solanum I. section Lycopersicon [Mill.] Wettst. subsection Lycopersicon). American Journal of Botany 88:1888-1902.

Peralta I. E. and D. M. Spooner (2005) Morphological characterization and relationships of wild tomatoes (Solanum L. sect. Lycopersicon) Monographs in Systematic Botany from the Missouri Botanical Garden 104:227-257.

Peralta I. E. and D. M. Spooner (2007) History, origin and early cultivation of tomato (Solanaceae). In: Genetic Improvement of Solanaceous Crops, Vol. 2: Tomato. M. K. Razdan and A. K. Mattoo (eds.). Science Publishers. Enfield, New Hampshire, USA. pp:124.

Peralta I. E., D. M. Spooner and S. Knapp (2008) Taxonomy of Wild Tomatoes and their Relatives (Solanum sect. Lycopersicoides, sect. Juglandifolia, and sect. Lycopersicon; Solanaceae). Systematic Botany Monographs Vol. 84. American Society of Plant Taxonomists. Ann Arbor, Michigan, USA. 186 p.

R Development Core Team (2012) R: A Language and Environment for Statistical Computing. R Foundation for Statistical Computing. Vienna, Austria. https://cran.r-project.org/doc/manuals/r-release/fullrefman.pdf. (Febrero 2017).

Rick C. M. (1983) Tomato (Lycopersicon). In: Isozymes in Plant Genetics and Breeding. Vol. 1, Part B. S. D. Tanksley and T. J. Orton (eds.). Elsevier Science Publishers. Amsterdam, The Netherlands. pp:147-165.

Rick C. M. and M. Holle (1990) Andean Lycopersicon esculentum var cerasiforme: genetic variation and its evolutionary significance. Economic Botany 44:69-78.

Rick C. M., J. W. De Verna and R. T. Chetelat (1990) Experimental introgression to the cultivated tomato from related wild nightshades. In: Horticultural Biotechnology: Proceedings of the Horticultural Biotechnology Symposium, held at the University of California, Davis, August 21-23, 1989. A. B. Bennett and S. D. O'Neill (eds.) Wiley-Liss. New York. pp:19-30.

Rick C. M. and R. T. Chetelat (1995) Utilization of related wild species for tomato improvement. Acta Horticulturae 412:21-38.

Ríos-Osorio 0., J. L. Chávez-Servia, J. C. Carrillo-Rodríguez y A. M. VeraGuzmán (2014) Variación agromorfológica y cambios biofísicos poscosecha en frutos de tomate (Solanum lycopersicum L.) Revista de la Facultad de Ciencias Agrarias. Unversidad Nacional de Cuyo 46:29-44

SAS Institute (2002) User's Guide of SAS (Statistical Analysis System). SAS Institute Inc. Cary, North Carolina, USA. 550 p.

Städler T., U. Arunyawat and W. Stephan (2008) Population genetics of speciation in two closely related wild tomatoes (Solanum Section Lycopersicon). Genetics 178:339-350

Steiner A. A. (1984) The universal nutrient solution. In: Proceedings of the 6th International Congress on Soilless Culture. 29 April - 5 May. International Society for Soilless Culture, ISOSC. Wageningen, The Netherlands. pp:633-650

UPOV, Unión Internacional para la Protección de las Obtenciones Vegetales 
(2011) Tomate (Solanum lycopersicum L.). Directrices para la ejecución del examen de distinción, la homogeneidad y la estabilidad. Documento TG/44/11. Unión Internacional para la
Protección de las Obtenciones Vegetales. Ginebra, Suiza. 70 p. Warnock S. J. (1988) A review of taxonomy and phylogeny of the genus Lycopersicon. HortScience 23:669-673. 